https://helda.helsinki.fi

\title{
How to Be a Humean Interventionist
}

\section{Kuorikoski, Jaakko}

2014-09

Kuorikoski , J 2014 , ' How to Be a Humean Interventionist ', Philosophy and

Phenomenological Research, vol. 89 , no. 2 , pp. 333-351 . https://doi.org/10.1111/phpr.12021

http://hdl.handle.net/10138/334305

https://doi.org/10.1111/phpr.12021

acceptedVersion

Downloaded from Helda, University of Helsinki institutional repository.

This is an electronic reprint of the original article.

This reprint may differ from the original in pagination and typographic detail.

Please cite the original version. 


\section{How to Be a Humean Interventionist}

\section{Jaakko Kuorikoski}

\section{University of Helsinki}

This paper aims to provide Humean metaphysics for the interventionist theory of causation. This is done by appealing to the hierarchical picture of causal relations as being realized by mechanisms, which in turn are identified with lower-level causal structures. The modal content of invariances at the lowest level of this hierarchy, at which mechanisms are reduced to strict natural laws, is then explained in terms of projectivism based on the best-system view of laws.

\section{Introduction}

The interventionist theory of causation has rapidly become the default picture of causal reasoning, especially in the philosophy of science. Yet many view the theory with suspicion because of its liberal use of primitive causal notions and because of the impression that the interventionist counterfactuals have not really been given proper truth conditions. In this view, the interventionist theory may well be useful in solving and disambiguating some conceptual puzzles. Yet because it takes too many conceptual and metaphysical liberties, it cannot be considered as a serious philosophical theory of what causation is. The proponents of the interventionist theory are quick to admit that the theory is indeed silent on matters of metaphysics (e.g., Woodward 2003, 121-122) and that the theory's strengths lay elsewhere. Yet concerns about the causal and modal primitives remain.

The purpose of this paper is to make the philosophical world safer for interventionists by showing that the interventionist theory and its associated counterfactuals can be given 
Humean regularity metaphysics, much in the spirit of David Lewis's Humean regularity metaphysics for his counterfactual analysis of causation. In order for the interventionist theory to make philosophical sense, we need to assume no more of the world than 'a vast mosaic of local matters of particular fact, just one little thing and then another'. (Lewis 1986, ix)

In essence, this essay is a foolhardy attempt at solving Hume's challenge of finding the secret 'connexion' between a cause and its effect. The methodology adopted here differs from many past philosophical attempts to solve this puzzle in that there will be little in terms of conceptual analysis. The methodology is premised on the view that conceptual analysis based on our intuitions should be distinguished from the metaphysical question of what the world should be like, in the most general terms, in order for it to inhabitable by beings with such concepts and intuitions. Here, the conceptual problem is considered already solved: our causal reasoning works, by and large, as characterized by the invariance under interventions account of Judea Pearl (2000) and James Woodward (2003).

Much of the philosophical literature on causality has proceeded by providing analyses of a single unique relation between two relata (be they objects, properties, events, or states of affairs) and testing these analyses against our intuitions about whether or not we would say that, in a given imaginary situation, a relation of causation obtains between the two imagined relata. Not surprisingly, no analysis immune to all the counter-examples presented has been put forward. By contrast, the interventionist theory of causation is not really an analysis of a single concept and thus does not provide a single unique relation for which metaphysics should be constructed. This is as it should be. Our causal reasoning simply does not work in such a way that a single analysis could be used to settle unambiguously whether the causal relation obtains between two arbitrary relata candidates of a suitable metaphysical category. The metaphysical work to be done is to tell a story about what kinds of things, in the most 
general sense, the world has to be made of in order for it to be inhabitable by cognitive agents using causal reasoning. The particular story to be told here will have recourse to the concept of mechanism, but not in a way that requires reference to causal or modal primitives. The story takes recourse only to fundamental regularities that characterize our world, and it needs only those modal notions, if any, that describe those regularities. ${ }^{1}$

Nevertheless, the metaphysics of causation should be objective in that the truth values of claims about causal relations do not depend on the particular person making them, and we (as an epistemic community) could well be collectively mistaken about particular causal facts. Again, this is simply taken as a premise. Most, if not all, science endeavors to uncover causal relations, and science is the final epistemic authority. If anything is to be taken as objective, then such claims surely should be. Our beliefs about causal dependencies in the world have undergone drastic historical changes, but these changes have certainly not altered whether particular causal claims are true or false. The task taken up here is to reconcile the requirement of objectivity of causation with the plurality of causal concepts and the contextdependence of causal intuitions on the one hand, and with Humean antirealism about causation on the other hand. My solution will turn out to be a mechanistic version of objective projectivism closely in line with the position Huw Price and Richard Corry call causal republicanism (Price and Corry 2007b), wherein causality is not a fundamental property of the world itself, but instead is a secondary quality definable only in relation to a possible perspective that divides the world into causal structures and their environments.

The argument proceeds as follows. The first section argues that the objective causal entity for which metaphysics should be given is neither the general causal law nor the token causal relation, but rather the token causal structure. The second section argues that a token causal structure is best characterized by using the invariance-under-interventions account of causation, considered here to be the correct description of causal reasoning. The third section 
argues that invariant dependencies constituting such structures are always realized by mechanisms, which in turn are simply further causal structures. The fourth section presents the core argument, in which this hierarchy of mechanisms is reduced to the level of fundamental physical regularities, and the modal content introduced on the lowest level of the hierarchy is explained as a projection arising from the introduction of the distinction between a system and its environment - between the inside and the outside. The fifth section discusses possible objections before offering some final thoughts.

\section{The primacy of a token causal structure}

The first step in this metaphysical project is the recognition that what a philosophical account of causal reasoning and, by implication, the metaphysics of causation should save is not the objectivity of claims about a privileged relation of causation, but the objectivity of a token causal structure. As Christopher Hitchcock has argued, the traditional philosophical project of providing an analysis of the causal relation has tried in vain to reach uniqueness and objectivity on the wrong level. The false presupposition has been that "In any concrete situation, there is an objective fact of the matter as to whether two events are in fact bound by the causal relation. It is the aim of philosophical inquiry to analyze this objective relation." (Hitchcock 2003, 4) First, there is no single relation of causation. Our causal reasoning is made up of multiple, related, but different causal concepts, such as contribution, net effect, relevance, dependence, production, direct cause, actual cause, prevention, and enabling. These concepts pick out relations of dependence with different formal properties and different levels of generality. Yet natural language does not always identify which specific causal notion is intended when something is said to cause something else, and thus it is no wonder that our intuitions sometimes are confused. The rival philosophical theories of causation are also usually based only on a subset of these notions, or at least, are especially 
good in dealing with such a subset. Probabilistic theories latch onto relevance or net effect, counterfactual theories latch onto dependence, process theories latch onto production, and so on. Yet it makes little sense to argue, at least with respect to many of these concepts, which of these is the one true relation of causation. (Hitchcock 2003)

Second, our intuitions concerning the relationship of token "causings" between two events - or what is nowadays more commonly referred to as "actual causation" - are especially context dependent to a degree that is disastrous for any hope of a single all-purpose analysis. This is a question of whether or not a token event is "the" cause of some other token event. Which causally relevant factors are the true causes and which are merely the enabling conditions? Was it Billy's or Suzy's throw that caused the bottle to break? Was it the poisoning of the water or the dehydration that caused the death of the desert traveler? Did the shooting of Archduke Ferdinand cause World War I? It seems that our intuitions concerning these kinds of cases are "polluted" by arguably non-causal considerations, such as what is the expected default state of the candidate relata, whether or not there is presence or execution of agency, how praise and blame are suppose to be attributed, and what kinds of interventions we should consider on prudent and ethical grounds. In many cases, these questions are relevant only for the diagnosis of malfunctioning mechanisms, the appropriation of praise or blame, or for purely intellectual amusement. These are not the kinds of considerations relevant to the metaphysics of causation. (Hitchcock 2007; Hitchcock and Knobe 2009; Knobe and Fraser 2008)

Nevertheless, Hitchcock points out that even when our intuitions or the judgments of rival philosophical theories diverge, there is a set of dependencies, a set of causal facts, on which the debaters can agree. These causal facts are not facts about general causal laws or about actual or token causation, the general ontological categories on which most metaphysical accounts of causation have focused. They are facts about a token causal 
structure, which is a set of causal dependencies instantiated in a spatiotemporally located individual, a token system (Hitchcock 2003; 2007). This is the causal entity for which metaphysics should be constructed. In the next section, I will argue that a token causal structure should be defined as a set of dependencies that are invariant under interventions.

\section{Why interventions?}

According to the interventionist account of causation (or causal reasoning), $\mathrm{X}$ is a cause of $\mathrm{Y}$ if and only if, by changing the value of $X$ through intervention, we could bring about a change in the value of Y. (Pearl 2000; Woodward 2003) Informally characterized, an intervention is an exogenously determined, ideal (causal) manipulation that directly changes only the target variable while leaving the rest of the causal structure intact. The concept of intervention guarantees that the dependency and the evaluation of the associated counterfactuals rely on an objective physical relationship between properties of objects, not on a purely epistemic or inferential relationship between our representations of these objects and their properties (this rules out, among other things, Lewis's backtracking counterfactuals). A variable taking a specific value at a specific time can, in standard fashion, be conceived as an event, thus preserving the generally accepted idea that singular (token) causal claims relate events, while maintaining that causal (relevance) relationships relate variables.

It is not possible to argue conclusively for the conceptual adequacy of the interventionist theory here, but a few points in its favor are in order. First, empirical psychological studies on actual causal cognition of humans (and some other animals as well) point to the importance of sequential manipulation of mental models in particular (e.g., Byrne 2002; Hegarty 2004; Schulz et. al. 2007) and of counterfactual reasoning in general. Of course, the way in which we actually reason about something is not the same thing as what it 
is to reason correctly about something, but surely the putative referent of the word "causation" has to have something to do with the way in we actually use the word.

A more weighty philosophical consideration in favor of the interventionist theory is that it is able to account for the intuitively plausible features of the other rival accounts of causation. Even though it is evidently not possible to provide a single analysis of the concept of the causal relation, the interventionist account demonstrates that there is a certain conceptual unity to causal reasoning. The Causal Markov condition provides a conceptual bridge between the causal structure and the conditional probabilistic dependencies, from which probabilistic theories of causation draw their intuitive plausibility. The interventionist theory is essentially a counterfactual theory, but it does not rely on possible world semantics, and its structural model semantics can be used to handle more complex counterfactual inferences (including probabilistic ones) than the standard Lewisian semantics. The concept of an active path formulated in structural models can account for the intuitions according to which, especially in token cases, a spatiotemporally continuous process exemplifies a "beefier" causal connection than mere counterfactual dependence, thus salvaging the intuitively appealing difference between production (process) and dependence. In short, it seems that the interventionist framework can deal with the totality of our causal reasoning practices, whereas other philosophical theories can, at most, handle only fragments.

Third, the close connection to manipulability provides a rationale for why cognitive agents such as us should be so interested in causal relations. Cognitive systems interested in manipulating and controlling their environment require knowledge of dependencies that are invariant under exogenous interventions, whereas purely passive observers interested only in non-manipulative prediction would be satisfied (and possibly better off) with mere regularities. The close conceptual link to manipulability also provides a direct connection to experimental practice: experimentation is the most direct way of examining causal relations 
because it is essentially a matter of applying something as close as possible to an ideal intervention in the system under investigation. The formal advances within the interventionist account have created an interdisciplinary research program linking statistics, computer sciences, and philosophy in a way that has also provided new statistical tools as well as much needed conceptual clarity to causal reasoning.

In a number of important respects the concepts of invariance and token causal structure differ from the standard philosophical concept of a natural law underpinning standard regularity accounts of causation. First, the (extensional) generality of a relationship does not, in principle, have anything to do with whether the relationship is causal. Second, invariances are change-relating and have to support a particular kind of (interventionist) counterfactuals. Third, exceptions do not render invariances non-causal, and this has important implications for what should be counted as evidence for, and against, causal claims. Fourth, more generally, investigating nested systems of causal structures (mechanisms) involves research heuristics that differ from those entailed by a nomothetic ideal of science. Therefore, even if some concept of lawhood were to prove indispensable to the metaphysics of causation in order for the metaphysical account to do justice to our practices of causal reasoning, the relationship between general laws and token causings cannot be a simple matter of a pair of events falling under a general causal law.

\section{A mechanistic theory of causation}

We have now argued that the metaphysics of causation should not provide us with a metaphysical account of the fundamental causal relation, revealed by conceptual analysis based on causal intuitions. Rather it should provide a story of how the world has to be built in order to be inhabited by agents engaged in causal reasoning as captured by the interventionist account and who are interested in the properties of token causal structures. The first part of 
this story is the acknowledgment that all causal invariances are realized by a mechanism. The idea that the concept of mechanism is an important ingredient in the theory of causality is by no means new. In a series of papers, Stuart Glennan $(1996 ; 2002 ; 2010)$ has argued that all causal relations are instances of "mechanically explicable laws." Also Stathis Psillos has suggested that mechanisms might offer us at least a glimpse of Hume's secret connection (2004).

The interventionist theory of causal reasoning is also based on the idea that causal dependencies rest on mechanisms. According to Pearl (2000), the structural models providing "the semantics" for interventionist counterfactuals represent mechanisms. The concept of mechanism is the default interpretation of functional dependency, which is the modal primitive of the interventionist account. The obvious next question is whether something more informative can be said about mechanisms, so that it makes philosophical sense to replace the primitive concept of 'causal relation' with that of mechanism. According to Glennan, a mechanism is "a system of direct, invariant, change-relating dependency relations (Glennan 2002)." In other words, a mechanism is simply a token causal structure. This view has recently been advocated by a number of other philosophers as well (Craver 2007; Kuorikoski 2012; Steel 2007; Woodward 2002). In this picture, the causal structure of the world is built on a hierarchy of mechanisms (Glennan 2010). Figure 1 illustrates the hierarchy of mechanisms: the invariant causal dependency between properties (variables) $a$ and $b$ is realized by a mechanism consisting of causal relations between the variables $\mathrm{X}_{1}, \mathrm{X}_{2}, \mathrm{X}_{3}$, and $\mathrm{Y}$. The causal relations mediating the influence of $\mathrm{X}_{1}$ and $\mathrm{X}_{2}$ on $\mathrm{b}$ are in turn realized by the mechanism consisting of further invariant dependencies between variables $\mathrm{Z}_{1}, \mathrm{Z}_{2}$, and $\mathrm{Z}_{3}$. 


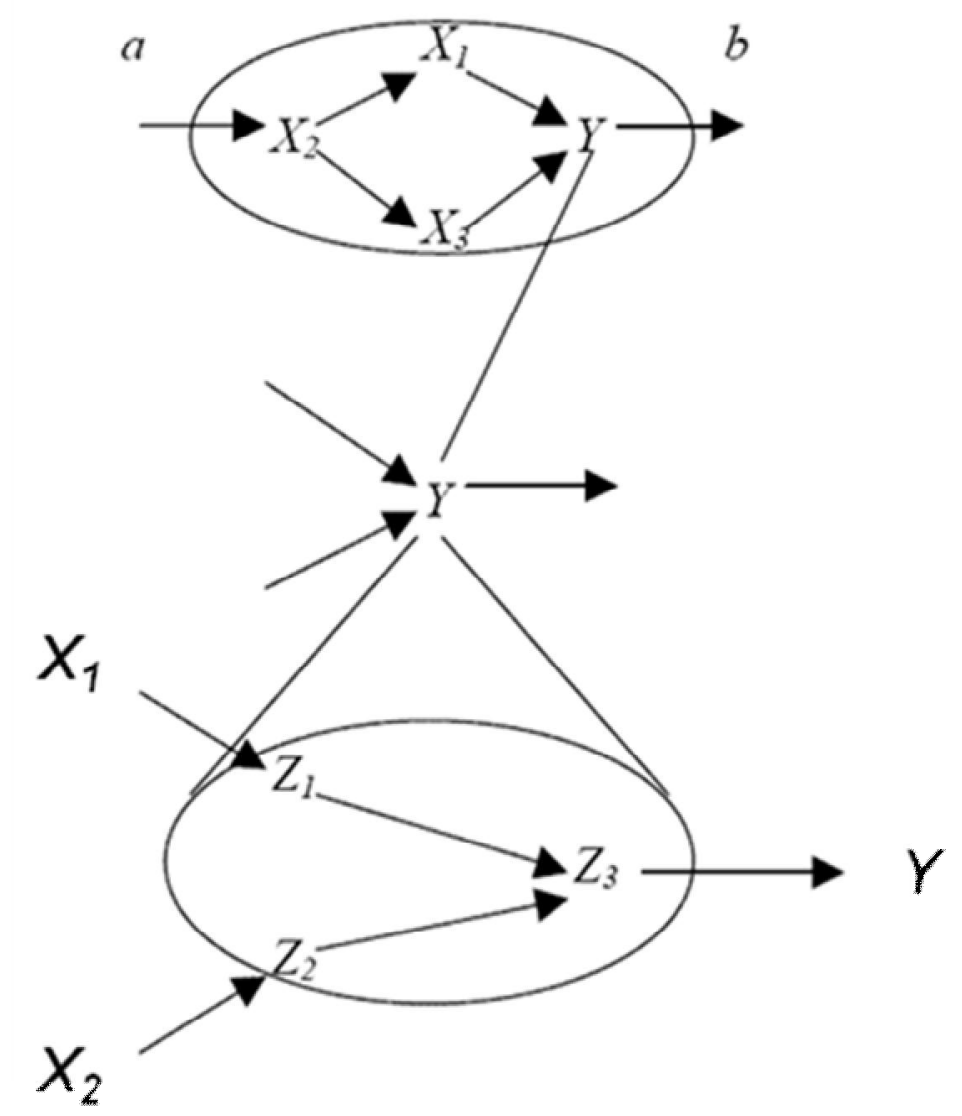

Figure 1. The hierarchy of mechanisms

One of the central questions immediately raised by the mechanistic approach to causality is whether it confuses epistemology with ontology, evidence with truth conditions. Obviously, finding out about mechanisms is an important way of acquiring knowledge about causal relations. But does this mean that causation is, in some deeper sense, simply a matter of the presence of a mechanism? I am certainly justified in asserting that, "If I were to turn the key, the car would start," if I know that there is a mechanism which connects key-turning with car starting (Glennan 1996). Yet how can the presence of a mechanism constitute the modal fact that if I were to turn the key, the car would start?

The hierarchical picture makes it clear that mechanisms can indeed provide proper truth conditions, and not just evidence, for interventionist counterfactuals. What makes it the 
case that had $X_{1}$ been set to value $x^{\prime}$ by an intervention, the value of Y would be $y$ '? Because setting $X_{1}=x^{\prime}$ leads to $Z_{1}=z_{1}$ ', which leads to $Z_{3}=z_{3}$ ' (given the value of $Z_{2}$ ), and this leads to $Y=y^{\prime}$. More generally, what determines that $Y=f\left(X_{1}, X_{3}\right)$ is invariant under interventions (and thus provides answers to counterfactual questions) is that $\mathrm{Z}_{1}=\mathrm{g}_{1}\left(\mathrm{X}_{1}\right), \mathrm{Z}_{2}=\mathrm{g}_{2}\left(\mathrm{X}_{2}\right), \mathrm{Z}_{3}=$ $\mathrm{g}_{3}\left(Z_{1}, Z_{2}\right)$, and $\mathrm{Y}=\mathrm{g}_{4}\left(\mathrm{Z}_{3}\right)$, and that all the dependencies $\mathrm{g}_{1} \ldots \mathrm{g}_{4}$ are also invariant under interventions. What makes $g_{1} \ldots g_{4}$ invariant is that all are realized by mechanisms, which, in turn, are just further causal structures providing the truth conditions for counterfactuals concerning interventions on the variables in $\mathrm{g}_{\mathrm{n}}{ }^{2}$ The common complaint - that the interventionist theory does not really provide semantics for the interventionist counterfactuals - is therefore somewhat misplaced, since in fact the semantics are directly provided by the modal primitive of a functional dependency between variables. ${ }^{3}$ It is just that philosophers expect the semantics of counterfactuals to look something like the possible world semantics, preferably extensional semantics (i.e., pairing names and values of variables with objects and predicates with sets) for propositionally structured content.

As an example, let us indulge in neuroscience. After all, Jack Smart famously quipped that causation is all right for plumbers and brain surgeons. Direct electrical stimulation of specific individual sites in the nucleus interpositus anterior in a cat cerebellum causes twitching in specific muscles of the cat. If one were to stimulate a cerebellar nuclear cell, a muscle would twitch. What realizes this dependency (and "makes" the associated interventionist counterfactuals true) is the mechanism running through the rubrospinal tract connecting the cerebellar nuclear cells to the muscle cells. This mechanism is constituted by invariant dependencies between activations in the nuclear cells, the motor neurons, the muscle cells, and the synapses between them. Intervening (stimulating) a nuclear cell causes an action potential to run through its axon, which causes a release of a neurotransmitter to the synapse, which then causes a post-synaptic action potential in the motor cell. What realizes 
the invariant dependency between the activation in a cell soma and the release of a neurotransmitter in the synapse is the mechanism of action potential. The action potential is a signal constituted by a series of invariant dependencies between the openings and closings of neighboring ion channels in the membrane of the axon and the resulting travel of sodium and potassium ions across the axon membrane. The channels that transmit sodium are voltage dependent (intervening on the surrounding voltage causes the channel to open or close), and thus, the local depolarization created by the inflow of sodium causes sodium channels to open further down the axon. What causes the sodium to enter through the opened channels are the electrical and concentration gradients between the inner and outer sides of the axon: intervene on the gradient and the movement of the ion changes. And what realizes the invariant dependency between the electrical gradient and ion movement? That is simply how charged particles behave according to the laws of physics.

Obviously, this way of fixing the truth-values of interventionist counterfactuals only passes the modal buck down the hierarchy of mechanisms. The apparent impossibility of deriving the modal "oomph" of causal powers of the component parts from something not having causal powers has been seen as the major obstacle to a completely reductionist mechanistic theory of causality (Glennan 2010; Psillos 2004). The task now is to locate the basis for this modal surplus and determine whether this can be done in a broadly Humean way. Is it really possible to pull a causal rabbit out of a Humean hat?

\section{Getting to the Bottom Rung of the Mechanistic Ladder}

According to Glennan's original mechanistic theory of causation, the mechanistic ladder bottoms out at the level of fundamental physical laws, and these physical laws are not causal laws. ${ }^{4}$ The idea is that causal reasoning is most at home in the special sciences, which investigate mechanistically explicable phenomena, whereas the proper domain of the concept 
of natural law is in fundamental physics. In the special sciences, observed regularities are phenomena to be explained by pointing out the underlying constellation of causal powers responsible for the regularity, whereas in physics, explanation truly amounts to the derivation of phenomena from more fundamental regularities, symmetries, and the like, as well as casespecific initial or boundary conditions. The view that causal concepts have little role in reasoning in fundamental physics (Russellian republicanism) has quietly become something approaching orthodoxy in the last ten years, at least in the philosophy of science (see the essays in Price and Corry 2007; for a contrasting view, see, e.g., Frisch 2009).

There are two key problems that this proposal needs to address. First, just saying that the ladder bottoms out at the level of physics or that the mechanisms at the lowest level "emerge" from physical laws amounts to no more than renaming the problem. We need an account of how and why this happens. What we need is an account of the (objective) truth conditions of active interventionist counterfactuals on the bottom rung of the mechanistic ladder where the modal buck can no longer be passed to a lower level of the mechanism. Second, if we want to pursue the Humean project, we need an account in which the fundamental physical laws are not characterized by a primitive natural necessity, the ability to support counterfactuals, or the like, which the causal relations could somehow simply inherit.

My suggestion is that the solution to these problems lies in a point made by Judea Pearl: causal concepts only make sense when a boundary between a system and its environment, between the exogenous and the endogenous, can be drawn. Causal reasoning is about the consequences of possible manipulations on a system and therefore makes sense only if there is an outside perspective from which these surgical interventions are contemplated. The crucial difference between causal/explanatory information and descriptive/predictive information is lost if there is no possibility or need to locally interrupt the endogenous dynamics of a system. As Pearl puts it, "If you wish to include the entire 
universe in the model, causality disappears because interventions disappear - the manipulator and the manipulated lose their distinction." (Pearl 2000, 349-350; see also Hitchcock 2007b, 53) According to this view, there is no causation in fundamental physics since the task of fundamental physics is to look at the world from sub specie aeternitatis. It tries to capture the most general regularities that characterize the world as a totality. Causal reasoning is introduced as soon as a physical system is conceptually isolated from the totality of being (the solar system, an atom, a particle), thus introducing the possibility of an outside intervention and the difference between endogenous dynamics and exogenous shocks.

But how can we get from this insight concerning the proper scope of causal reasoning to providing objective truth conditions for interventionist counterfactuals? Let us take a simple two-dimensional Hume world represented in Figure 2.

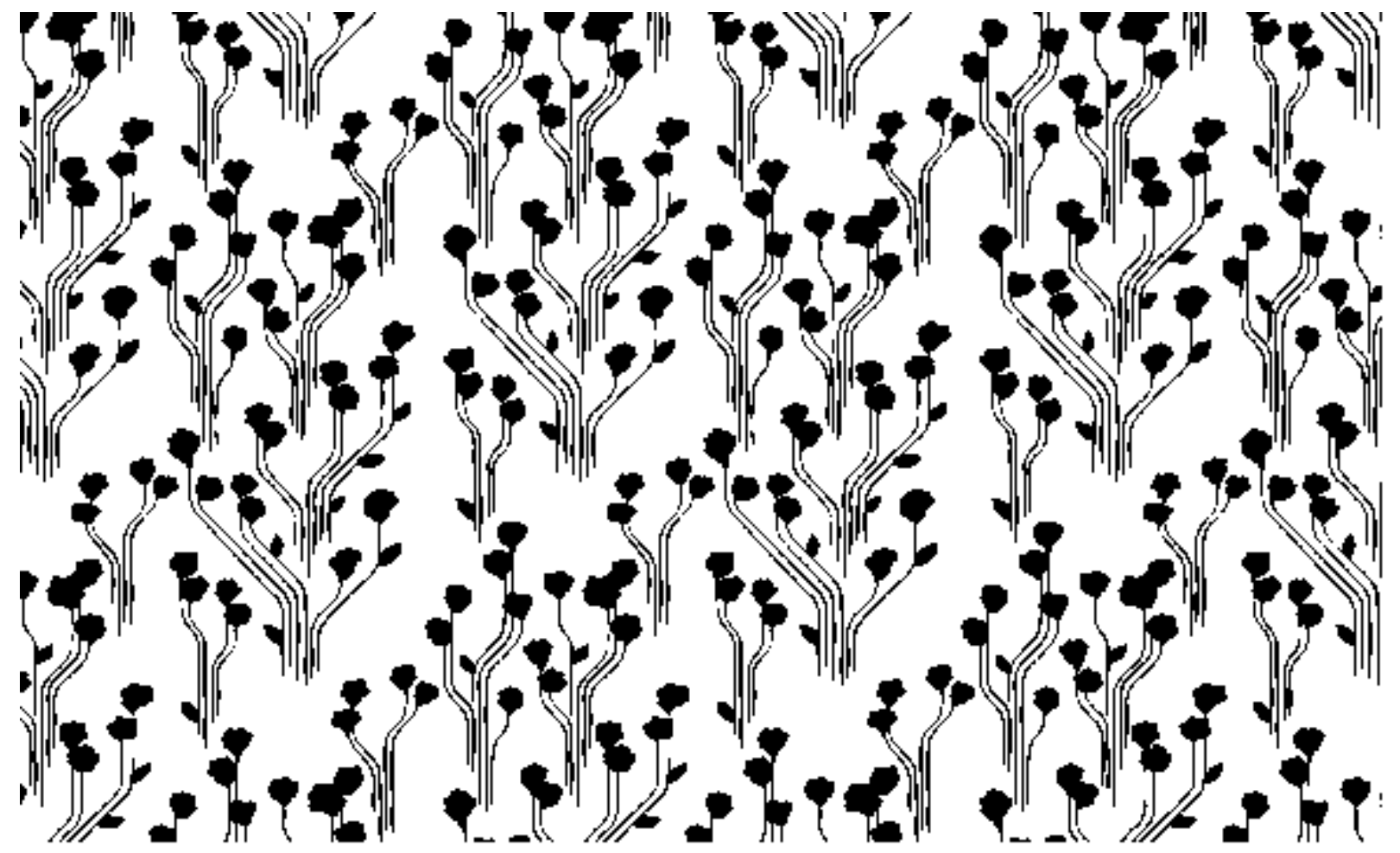

Figure 2. A two-dimensional Hume world. 
This world is not very interesting and certainly not very pretty. Figure 2 represents all there is in the Hume world: two properties (black and white) and two dimensions. ${ }^{5}$ One can think of the $\mathrm{y}$-axis as time and $\mathrm{x}$-axis as a singular spatial dimension, but, strictly speaking, such attributions are only projections from our outside perspective and not intrinsic properties of the Hume world. The world is strictly Humean in that it does not have any carriers of natural necessity: it just is. Just one little thing and then another. Nevertheless, it is most certainly regular. The whole of the Hume world can be described by a finite set of descriptions of regularities, and the most parsimonious of such axiomatizations can be said to define the Mill-Ramsey-Lewis laws of the Hume world. The MRL laws are the laws of physics for the Hume world. If we take the $y$-axis (or the $\mathrm{x}$-axis for that matter) to represent time, these MRL laws allow us to predict (and retrodict) what will happen (or has happened), given an accurate description of only a local area of the Hume world.

Now let us separate part of the Hume world to be a system, thus dividing the world into an inside and an outside (Figure 3). 


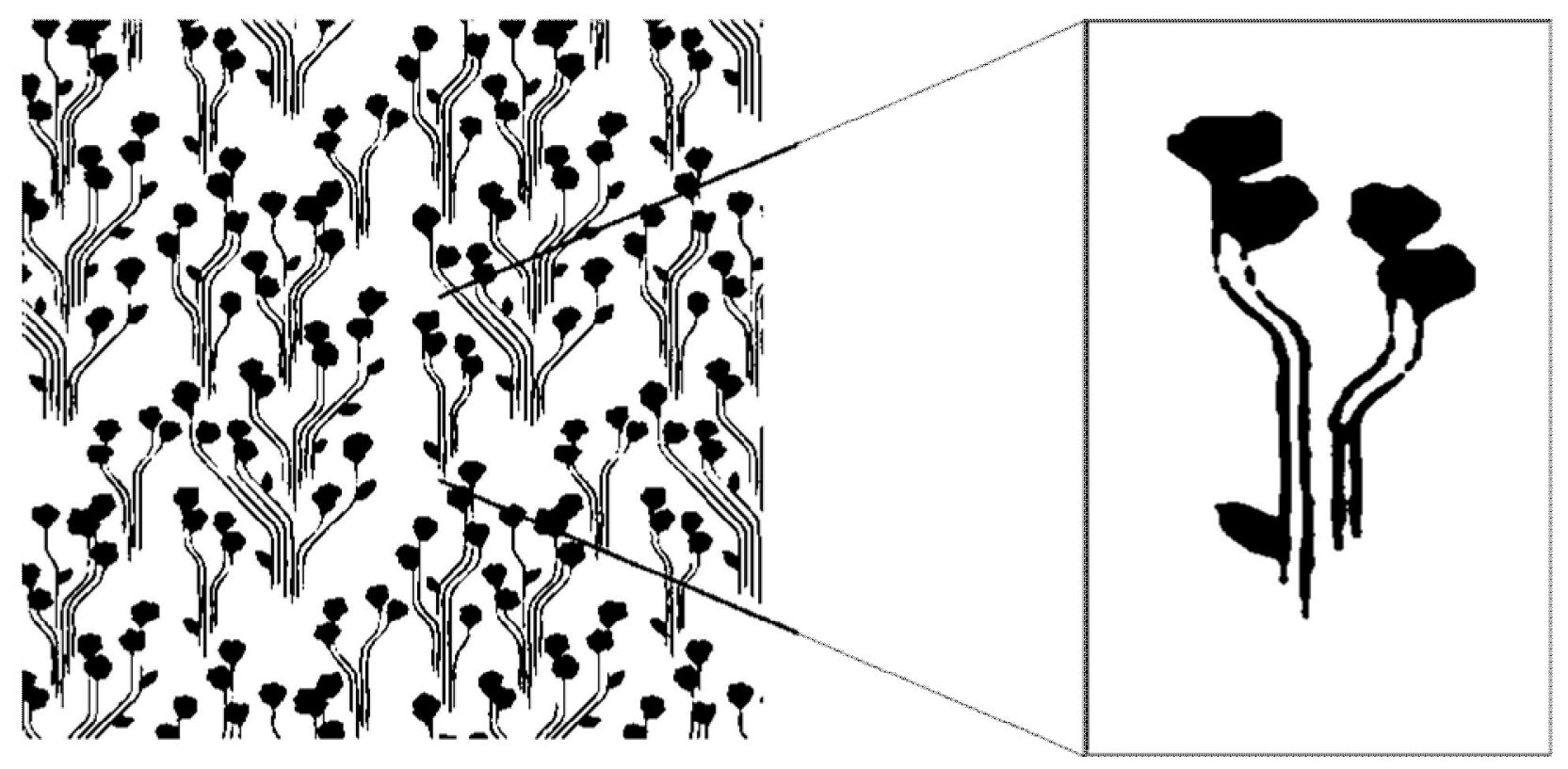

Figure 3. A system and its environment.

Now that we have an inside and an outside, we can contemplate the consequences of a local intervention. Suppose that an agent (for the moment, let us set aside the question of the agent's location) is trying to predict the consequences of the following local manipulation:
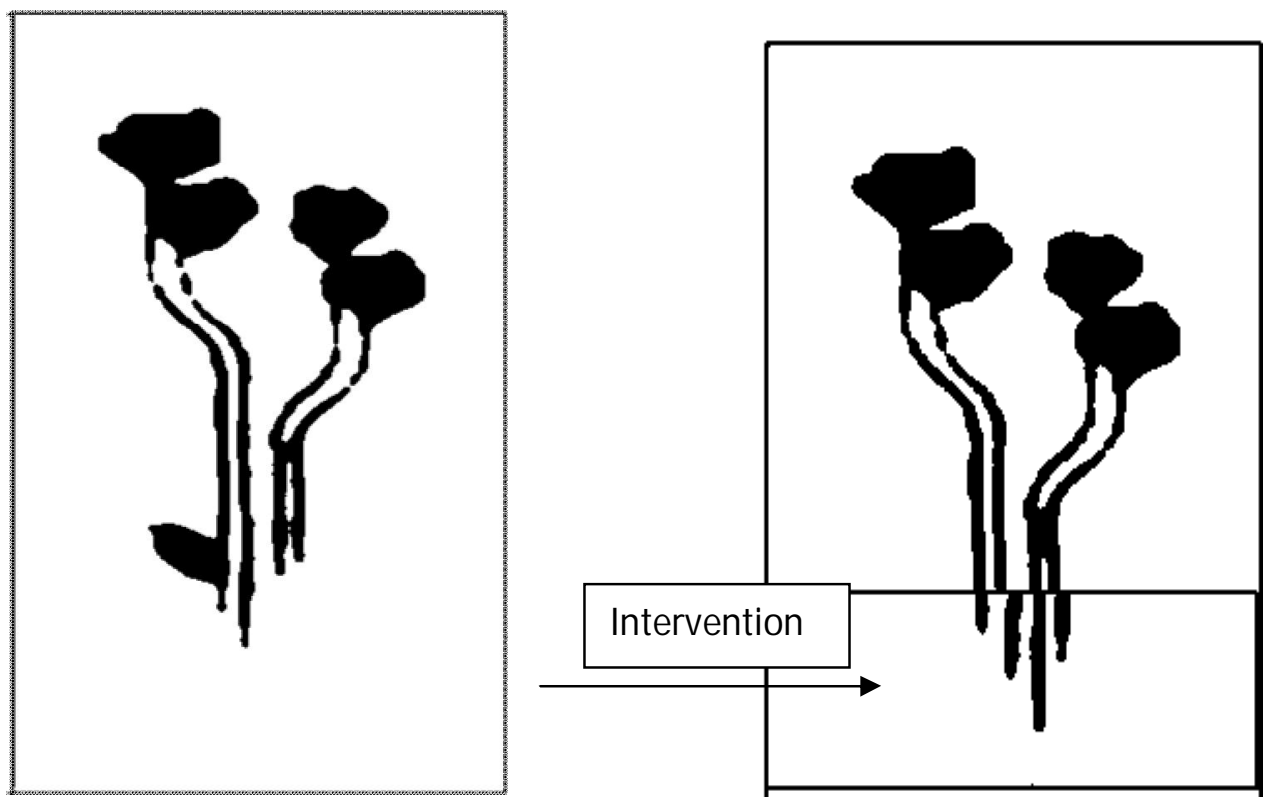

Figure 4. A local intervention.

Arguably, the rational thing for the agent to do is to use the MRL laws to predict the consequences of this local intervention. 

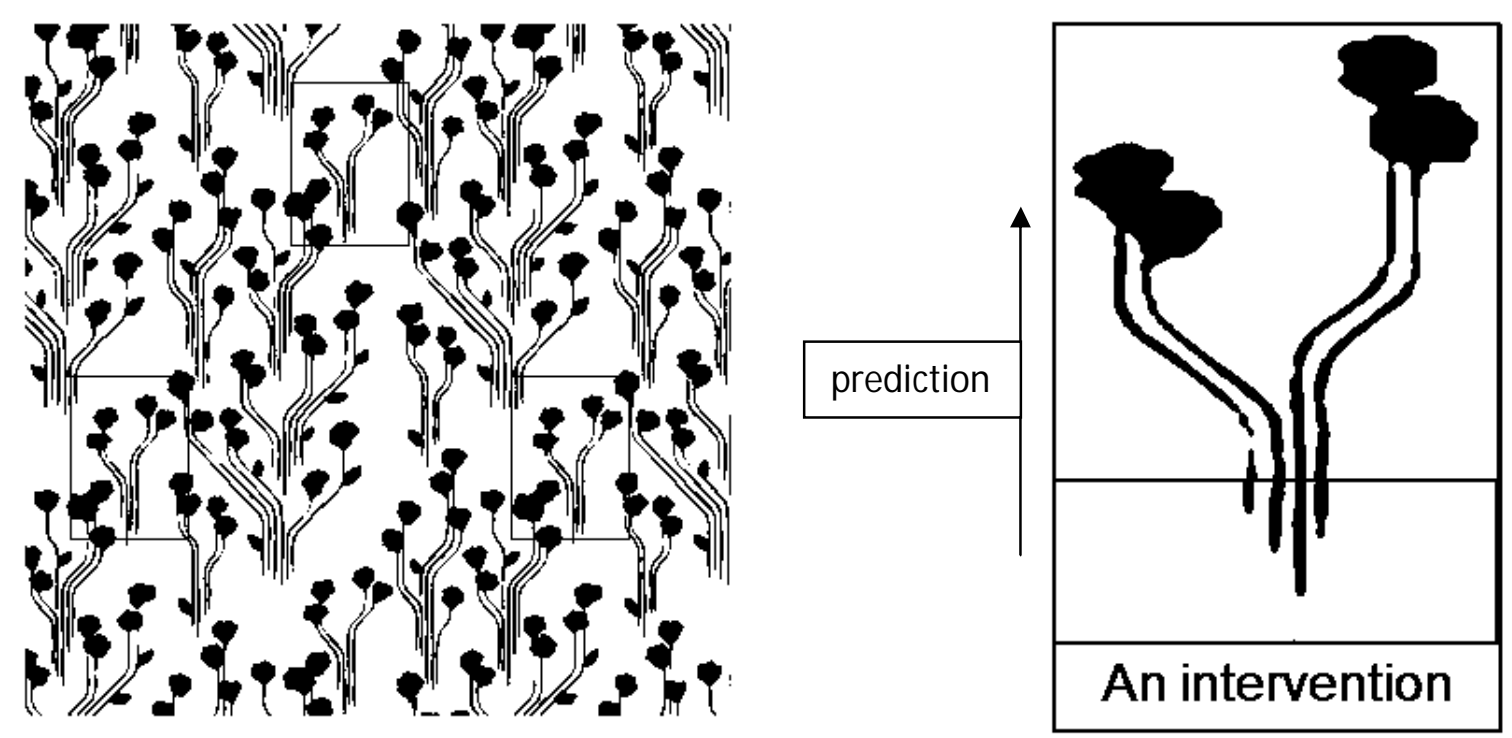

Figure 5. The consequences of an intervention derived from the Mill-Ramsey-Lewis laws.

At this point the reader is doubtless vigorously shaking a finger and shouting that it is completely arbitrary simply to stipulate that the use of the MRL laws to predict the consequences of the intervention is unambiguously rational and that the introduction of an agent into the Hume world is already "breaking the rules" of this little metaphysical modeling exercise. After all, there was no mention of any agents inhabiting the Hume world. And even if some argument could be given in favor of these moves, this account can at most provide assertability conditions for interventionist counterfactuals, not properly "objective" truth conditions.

But the agent need not reside on the outside the Hume world, no more than we are outside our world. If and when the agent is actually part of the fabric of the Hume world, the intervention, when carried out, is also part of the Hume world, and through the assumption of orderliness, also "governed" by the MRL laws of the Hume world. After all, our own interventions in the actual world are always "governed" by the laws of physics. The word 'governed' should be read as metaphorical: there is no natural necessitation involved in the congruence of the agent, intervention, consequences, and the MRL laws; the congruence is 
just a consequence of the brute fact of the matter that the Hume world is orderly. From the sub specie aeternitatis viewpoint by which the MRL laws are defined, all interventions are endogenous.

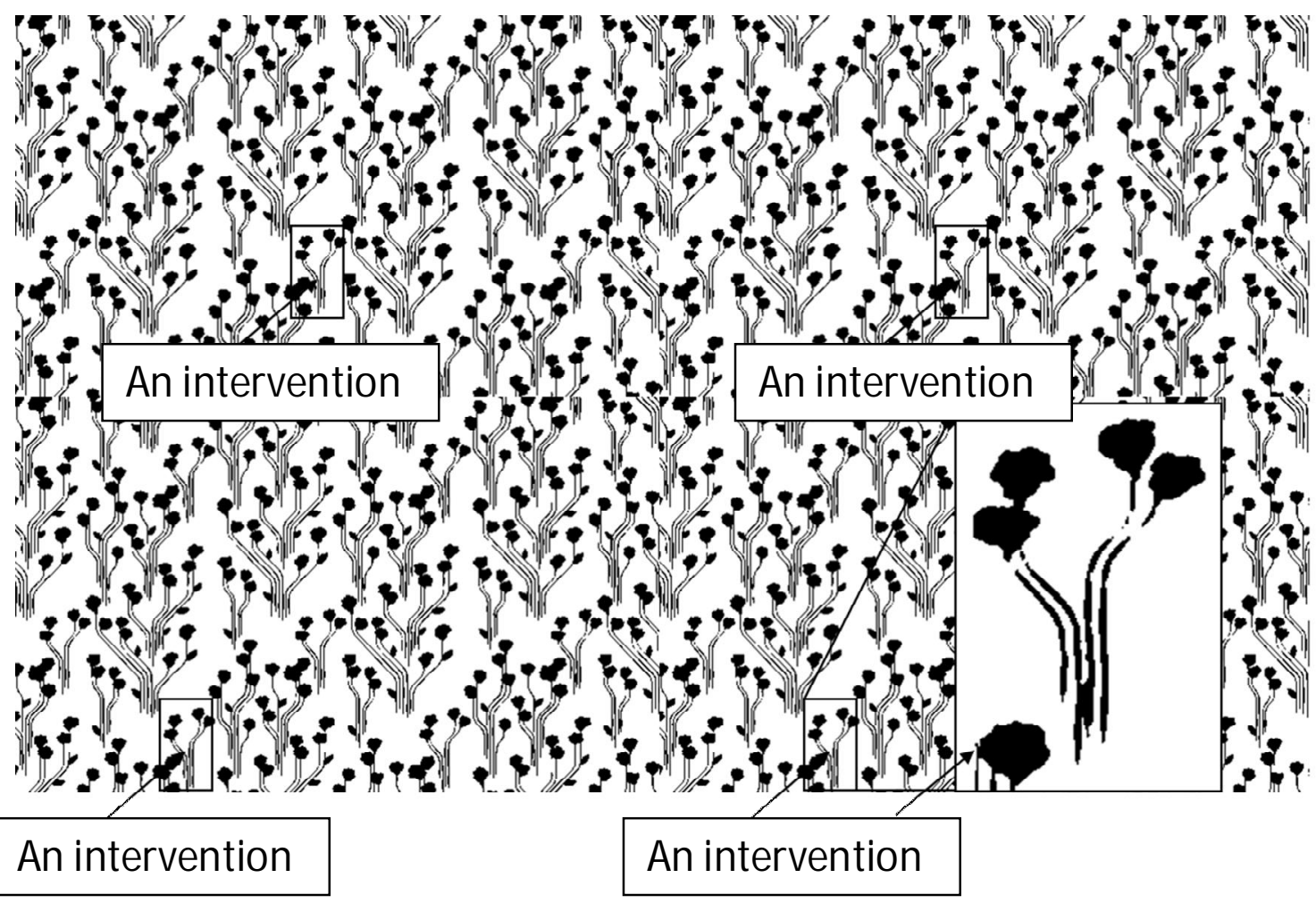

Figure 6. Placing the manipulator into the Hume world.

Thus, from the point of view of any arbitrary manipulator within the Hume world, it is a completely objective fact that if a system of a kind shown in Figure 3 is intervened on as in Figure 4, the result will be as seen in Figure 5. Again, from the point of view of any arbitrary manipulator, if a particular system, which in actuality is not intervened on, had been thus intervened on, the consequence would have been what is "dictated" by the MRL laws. But this is only because in such a situation, the manipulator would also have been part of a slightly different pattern of events, a pattern consistent with the intervened state of the system and the MRL laws. From a local point of view in which there is a distinction between a system and its environment, there are objective truth conditions for interventionist counterfactuals. But from sub specie aeternitatis without the endogenous-exogenous 
distinction, there is only a pattern of non-intervening manipulators and corresponding nonintervened systems on the one hand, and intervening manipulators and corresponding intervened systems on the other hand.

The above reasoning sheds some light on a much discussed feature of the interventionist theory, namely that the very definition of intervention makes use of causal concepts and that the theory is not, and indeed cannot be, a reductive theory of causation because of this. For example, interventions have to break the causal connections between the target variable and its causal parents and an intervention cannot be a direct cause of any other variable, and such criteria cannot be adequately formulated in purely associational terms. In a sense, this is to be expected, since the aim of the theory is to regiment causal reasoning and the work done by the intervention concept in such regimentation already presupposes the inside/outside distinction. In contrast, from sub specie aeternitatis interventions are not characterized by any specific pattern of events. They are just sequences of events like any other. They can be distinguished as causal interventions only from a local perspective, from which they are not seen as a part of the endogenous dynamics of some subset of the Humean fabric considered in isolation, but as an outside influence entering the subset from the outside and severing the associations within it in an appropriate way. But if we look at the big (total) picture, this entering and severing is just a part of the general orderly pattern of events summarized in the MRL laws.

When an ion channel opens in the axon membrane, a sodium ion moves into the axon along the electric gradient. If one were to intervene on the gradient or (rather miraculously) on the charge of the particle, the movement would change. But what makes this subjunctive conditional true, since presumably there is no further underlying causal structure realizing this invariant dependency? The electric gradient is there because of the other negatively charged ions within the axon. Considered in isolation, describing the movement of two 
oppositely charged particles does not require any causal vocabulary; it is simply how certain kinds of things behave, always and everywhere. In fact, it makes little sense to claim that the negative charge causes the movement of the positively charged particle or vice versa. As Russell observed, the laws of electrodynamics are symmetrical and only provide the trajectories of particles given the initial conditions. But if one considers intervening on the two-particle system from the outside, for example, by introducing a second positively charged particle, the laws of physics also seem to dictate what would happen in such a case. But this is just because they state how, under such initial conditions that are consistent with the contemplated intervention, such three-particle systems always, in fact, behave. The subjunctive tense is only relevant from the outside perspective. And if a third particle is in fact introduced, the introduction event also "follows" the laws of physics, i.e., from sub specie aeternitatis, the actual intervention is simply part of the pattern of regular events as summarized in the MLR laws. This concludes the explication of the truth-conditions of interventionist counterfactuals on the bottom rung of the mechanistic ladder.

\section{Replies to possible objections}

Causal facts are local. What happens in Andromeda has no bearing on whether or not a relation between two variables here on earth is causal. Therefore, the MRL laws cannot be the truth-makers for causal claims. Causal facts indeed are local facts, and the truth-makers for interventionist "same-object" counterfactuals (Woodward 2003, 281) are the token mechanisms realizing the corresponding causal dependencies, which are very much "local." But if one finds counterintuitive the very idea that what happens in Andromeda is relevant to what the laws of physics are (qua MRL laws), then that is a problem not only for the account of causation given here, but also for the best-system view of laws. Such concerns cannot be addressed here. 
A Humean stance on laws of nature commits one to the principle of plentitude, to a view that it is simply a fluke that the universe doesn't fall apart the next second, and to a host of other modal absurdities. In principle, the argument given here is only committed to the claim that the modal content of causal claims is not directly inherited from any possible primitive natural necessity possessed by the laws of nature. There may be independent metaphysical reasons not to accept a fully Humean view of the laws of nature, but whatever natural necessitation has for these reasons to be recognized, it is not the same thing as the "necessitation" of causation.

But it demonstrably follows from the interventionist theory itself that causation cannot be reduced to regularities. Indeed, it is only in exceptional cases that causal structures are uniquely identifiable from passive observations of covariances (see below) and in this sense causal structures do not reduce to regularities. Yet if the story told above is true, then causal structures as such are definable only in relation to the larger whole in which they are embedded, and they reduce to regularities only when the world (the whole of space-time) is viewed in its totality. Causality thus "reduces" to regularities only at the limit, when the possibility of causal reasoning itself disappears. The formal interventionist theory of causal reasoning is only meant to apply to finite structures, and thus already presupposes the inside/outside distinction. Therefore any specific results derived from it have little bearing on the plausibility of the global reduction.

To see the point more clearly, consider an important special case in which a causal structure is uniquely identifiable from passive observations alone. This is the case when each and every endogenous variable has its own observable cause, which itself is independent of any other variable in the structure. It is also the case that every (causally sufficient) structure could in principle be rendered identifiable by embedding it in such a structure (Spirtes, Glymour and Scheines 2000, 65). This would effectively amount to providing each 
endogenous variable with its own independent source of variation. It is therefore an interesting question whether such identifiability results could provide a more refined reduction of causal structures to only restricted subsets of the global Humean regularities. The idea would be that the truth conditions of interventionist counterfactuals could be fixed by the occurrent regularities in those cases in which there actually happens to be such an independent source of variation for the cause variable. ${ }^{6}$ Although trying to find such variables is obviously an effective epistemic strategy for discovering causal relationships (think of instrumental variable estimation and natural experiments), it is nevertheless not a way of grounding the truth values of causal claims. The above result about the in principle recoverability by provision of additional variables is true for all causal structures at all levels of mechanism, but the Humean reduction happens only at the "bottom level" where the mechanisms are directly constituted by fundamental regularities. In all but the lowest levels of mechanism, the truth values of interventionist counterfactuals are determined by the local mechanism realizing the causal dependency in question and the particular constellation of fundamental regularities that realizes a particular mechanism "at the bottom level" may very well be unique. So even if there is only one instance of a particular invariance in the universe, and consequently no other similar relations with additional independent exogenous causes that would help in its identification as causal, related interventionist counterfactuals would still have truth values. It is telling that the identifiability result itself is not formulated in purely associational terms: the supplementary observable independent variables guaranteeing identifiability are causes of the endogenous variables, not just correlated with them. So although the causality of any local structure cannot be exhaustively reduced to only local Humean regularities, this does not mean that causation would be irreducible when the world is viewed in its totality. 


\section{Concluding thoughts}

At this point it should be kept in mind why we cannot simply dispense with the whole story of the mechanistic hierarchy and live with a simpler projectivist account, in which causal relations, even in the sense of the interventionist account, are pairs of events falling under an instantiation of an MRL law, or something similar (as in Cohen and Callander 2010). We cannot jump straight from laws to causes, because what we were after was the metaphysics of a token causal structure, which, in all but the limiting lowest-level cases, are always locally realized by mechanisms - not regularities. Even though mechanisms metaphysically bottom out to brute regularities, our causal reasoning - as described in the interventionist theory and realized in scientific practice - is about dependencies realized by mechanisms - not regularities. We are rarely in a position to evaluate the effectiveness of our interventions directly on the basis of the MRL laws of our world, i.e. the laws of physics. We reason about local, structure-specific dependencies, not law-like regularities, and use manipulative experiments and knowledge of lower-level dependencies as evidence for these dependencies. Hence, we need the more complicated story presented here: what is objective is the token causal structure, made up of dependencies that are invariant under interventions. Such dependencies are realized by mechanisms, which in turn are just structures of further invariances. At the lowest level of this hierarchy, in which the mechanisms are directly constituted by fundamental laws, the "modal force" of causation is a projection arising from a perspective that isolates the mechanism as a system distinct from the universal regularities in which it is embedded, thus creating a distinction between the inside and the outside. From this restricted perspective, the truth conditions of interventionist counterfactuals are objectively determined by the MRL laws of the world. Thus, causation is an objective, but not irreducibly primitive, property of the world when seen in its totality sub specie aeternitatis. ${ }^{7}$ 


\section{References:}

Beebee, Helen. 2006. “Does Anything Hold the Universe Together?” Synthese 149: 509-533.

Cohen, Jonathan and Craig Callender. 2010. "Special Sciences, Conspiracy and the Better

Best System Account of Lawhood.” Erkenntnis 73:427-447.

Craver, Carl. 2007. Explaining the Brain. Oxford University Press.

Glennan, Stuart. 1996. "Mechanisms and the Nature of Causation.” Erkenntnis 44 (1):49--71

Glennan, Stuart. 2002. "Rethinking Mechanistic Explanation.” Proceedings of the Philosophy of Science Association 2002 (3):S342-353.

Glennan, Stuart. 2010. "Mechanisms, Causes, and the Layered Model of the World."

Philosophy and Phenomenological Research 81 (2):362-381.

Frisch, Mathias. 2009. “'The Most Sacred Tenet'? Causal Reasoning in Physics.” British Journal for the Philosophy of Science 60 (3):459-474.

Hegarty, Mary. 2004. "Mechanical reasoning as mental simulation." Trends in Cognitive Science 8: 280-285.

Hitchcock, Christopher. 2003. "Of Humean Bondage.” British Journal for the Philosophy of Science 54 (1):1-25.

Hitchcock, Christopher. 2007a. “Three Concepts of Causation.” Philosophy Compass 2 (3):508-516.

Hitchcock, Christopher. 2007b. "What Russell Got Right.” in Price and Corry (eds.): 45-65. Hitchcock, Christopher and Joshua Knobe. 2009. "Cause and Norm.” Journal of Philosophy $106(11): 587-612$.

Knobe, Joshua and Ben Fraser. 2008. "Causal Judgment and Moral Judgment: Two Experiments.” In Walter Sinnott-Armstrong (ed.), Moral Psychology. The MIT Press. 
Kuorikoski, Jaakko. 2012. "Mechanisms, Modularity and Constitutive Explanation." Erkenntnis 77(3): 361-380.

Lewis, David. 1986. Philosophical Papers Volume II. Oxford University Press.

Machamer, Peter, Lindley Darden and Carl Craver. 2000. “Thinking About Mechanisms.”

Philosophy Of Science 67 (1):1-25.

Pearl, Judea. 2000. Causality - Models, Reasoning and Inference. Cambridge University Press.

Price, Huw and Richard Corry. (eds.) 2007. Causation, Physics, and the Constitution of Reality: Russell's Republic Revisited. Oxford University Press.

Price, Huw and Richard Corry. 2007b. “A Case for Causal Republicanism?” In Huw Price \& Richard Corry (eds.).

Psillos, Stathis. 2004. “A Glimpse of the Secret Connexion: Harmonizing Mechanisms with Counterfactuals.” Perspectives on Science 12, 288-313.

Psillos, Stathis. 2006. “What Do Powers Do When They Are Not Manifested?” Philosophy and Phenomenological Research 72: 137-156.

Schulz, Laura, Alison Gopnik, and Clark Glymour. 2007. "Preschool children learn about causal structure from conditional interventions.” Developmental Science 10: 322-332.

Spirtes, Peter, Clark Glymour and Richard Scheines. 2000. Causation, Prediction and Search ( $2^{\text {nd }}$ edition). The MIT Press.

Steel, Daniel. 2007. Across the Boundaries: Extrapolation in Biology and Social Science.

Oxford University Press.

Strawson, Galen. 1991. “The Contingent Reality of Natural Necessity.” Analysis 51, 209-213. Woodward, James. 2002. "What is a Mechanism? A Counterfactual Account." Philosophy of Science 69: S366-S377. 
Woodward, James. 2003. Making Things Happen - A Theory of Causal Explanation. Oxford University Press.

${ }^{1}$ Hence, I leave it open as to whether there is a conceptual possibility or even an explanatory need for something by reason of which the world is regular (Strawson 1991), something “holding the universe together." However, Helen Beebee's arguments against such a need are persuasive (Beebee 2006).

${ }^{2}$ This cuts some very big corners, because there is also the modally loaded question of why the functional forms are as they are. The functional forms (and parameters) depend constitutively on the properties of the lower- level invariances, and this relation of constitution is also a counterfactual dependence, but not, strictly speaking, causal (Kuorikoski 2012). Hence, this dependency would require a separate Humean treatment. Variables on different levels of a mechanism can also be related in a more complicated manner than in this simple case.

${ }^{3}$ It is also important to note that when Pearl is providing "semantics for counterfactuals" with his abduction-intervention-prediction procedure (e.g., Pearl 2000, 37), he is talking about more complex counterfactual expressions than those handled by Lewis, for example. This procedure is built upon the more primitive, "direct" semantics of functional dependencies. Sometimes philosophers mistake this more elaborate account as an alternative theory for the semantics of counterfactuals in general. 
${ }^{4}$ By contrast, in their seminal paper on mechanisms, Machamer, Darden, and Craver (2000) state that the level on which mechanisms "bottom out" is always relative to the field and object of study.

${ }^{5}$ That the idea of pure non-dispositional properties makes sense to begin with (and that pandispositionalism is not a conceptual truth) is here simply taken as a premise (see, e.g., Psillos 2006).

${ }^{6}$ This idea was suggested to me by an anonymous referee.

${ }^{7}$ Earlier versions of this paper have been presented at the European Congress of Analytical Philosophy 6, at IHPST workshop on Counterfactuals and Mechanisms at Ecole Normale Superieure in 2011, and in the Philosophy of Science seminar at the University of Helsinki. I thank the participants for their valuable input. I am especially grateful to Marion Godman for her help with some finishing touches and to Carl Craver for pushing me to answer the questions in section 5. This research has been supported by the Academy of Finland. 